\title{
El proceso colectivo ambiental: una caracterización sui generis
}

\section{POR MATÍAS OSCAR MUÑOZ(*)}

Sumario: I. Los procesos colectivos en general.- II. Acciones de clase y proceso ambiental.- III. Los precedentes de la Corte argentina y las facultades del juez.- IV. El caso España: un ejemplo comparado.- V. Conclusión.- VI. Bibliografía.

Resumen: los procesos colectivos en Argentina cuentan con determinadas reglas que la Corte Suprema de Justicia de la Nación ha delineado tanto en sus acordadas como en sus sentencias, debido a la mora legislativa en reglamentar estos procesos por parte del Congreso Nacional. El proceso ambiental aparece como un caso particular, disociado de la acción de clase adaptada al derecho argentino, en el que el máximo tribunal reconoce en el juez ciertas herramientas exorbitantes en virtud del bien jurídico protegido. Este aspecto parece abrir camino a un proceso sui generis, con sus propias reglas pretorianas que la Corte reitera a los jueces de grado en sus casos emblemáticos.

Palabras clave: procesos colectivos - acciones de clase - ambiente

(*) Abogado. Esp. en Derecho Ambiental y Doctorando, Universidad de Buenos Aires (UBA). Prof. Adjunto de la Universidad Argentina de la Empresa (UADE). Prof. Adjunto de la Universidad de Ciencias Sociales y Empresariales (UCES). Jefe de Trabajos Prácticos de la Universidad de Buenos Aires (UBA). Investigador del Instituto de Ciencias Sociales y Proyectuales (INSOD-UADE). Investigador del Instituto Lucas Ambrosio Gioja (UBA). Dir. de Proyecto de Investigación (UADE). Miembro de Proyecto de Investigación (UBACYT 2018; Binacional CONICET 2018). 


\section{The collective environmental process: a sui generis characterization}

Abstract: the collective processes in Argentina have established rules that the Supreme Court of Justice of Argentina has outlined both in its agreements and in its judgments, due to the legislative delay in regulating these processes by the National Congress. The environmental process appears as a particular case, disassociated from the class action adapted to Argentine law, in which the highest court recognizes in the judge certain exorbitant tools under the protected legal right. This aspect seems to open the way to a sui generis process, with its own Praetorian rules that the Court reiterates to the judges of degree in their leading cases.

Keywords: collective processes - class actions - environment

\section{Los procesos colectivos en general}

Los procesos colectivos - también denominados acciones colectivas, acciones de clase, etc. - tienen una breve pero intensa tradición en el derecho argentino. En efecto y si bien la Constitución reformada en el año 1994 trajo aparejada la recepción explícita de ciertas mutaciones ocurridas con carácter previo a su modificación, lo cierto es que el campo de los derechos colectivos (con especial énfasis en materia ambiental) el gran debate jurisprudencial -en todas las instancias jurisdiccionales- ocurría en cuanto a la legitimación de uno o varios sujetos arrogándose la representación de otros o bien respecto a la defensa de bienes colectivos.

En particular, en el campo del derecho ambiental, los precedentes (1) fueron haciendo lugar a pretensiones invocadas en defensa del ambiente, en un tiempo en el cual afloraba la materia en virtud de las Conferencias Internacionales (Estocolmo 1972 y las subsiguientes celebradas en Río de Janeiro 1992, 2002, 2012 respectivamente). Es importante señalar que el precitado "Kattan", uno de los más novedosos precedentes de aquel momento (en el cual el ambiente aún no tenía la preponderancia actual, pero comenzaba a ganar territorio) hace explícita referencia a Estocolmo:

En la Argentina tenemos una ley (22.421), que protege la fauna silvestre; esta normativa sigue el espíritu de la Conferencia de Estocolmo (P. N. U. M. A. 1972), pero aquí, como en otros lugares del mundo, las leyes, por un motivo u otro, no se han puesto en práctica; las Convenciones no se ratifican; los organismos no cuentan con fondos suficientes para llevar a cabo sus altruistas fines. Puede decirse que en muchas partes del mundo no se es consciente de la gravedad del problema,

(1) CNFed. Cont. Adm., sala III, 08/09/1994, in re, "Schroder, Juan c. Estado Nacional - Secretaría de Recursos Naturales"; Juzg. Nac. en lo Cont. Adm. Federal, “Kattan”, 10/05/1983, La Ley, 1983-D, 576 , entre muchos otros. 
sea por desconocimiento, negligencia o irresponsabilidad (ver Declaración de Organizaciones no Gubernamentales en Sesión de Carácter Especial del Consejo de Administración del P. N. U. M. A. Programa de las Naciones Unidas para el Medio Ambiente-Nairobi, mayo de 1982) (Fallo citado, Considerando IV).

En otro orden de cosas, en el año 1993 se dictaba la Ley de defensa del consumidor No 24.240. Este estatuto se asomaba tímidamente en el ámbito del derecho privado y su carácter de orden público (conforme artículo 65) no tuvo lugar hasta entrada de la década del 2000. En el año 2008 la ley No 26.361 incorporó las acciones de incidencia colectiva (artículo 27 de la ley; actual artículo 54 de la ley 24.240), señalando el carácter erga omnes de la sentencia y permitiendo el opt out para aquellos usuarios o consumidores que con carácter previo al dictado de la sentencia manifiesten su voluntad en contrario (artículo 54, segundo párrafo ley 24.240).

Los dos pilares entonces del derecho colectivo serían, por un lado, el ambiente (bien colectivo indivisible) y el derecho de los consumidores y usuarios (afectación a grupo o clase, bien colectivo divisible). En torno a ellos la Corte señalaría más tarde la existencia de dos procesos: un proceso referido a bienes colectivos ( $v . g r$. ambiente) y un proceso con cierta similitud a la acción de clase norteamericana denominado proceso colectivo "referido a intereses individuales homogéneos".

Es menester señalar que los derechos de incidencia colectiva suponen un catálogo abierto. Es decir, si bien los dos pilares arriba señalados suelen ocupan gran parte del repertorio jurisprudencial, lo cierto es que siempre pueden surgir nuevas manifestaciones de derechos colectivos, como lo fue la discriminación racial o étnica en la class action norteamericana del siglo pasado. En nuestro país - por dar un ejemplo- el derecho previsional es fuente de procesos colectivos.

En este tipo de acciones, si se configura el colectivo o clase, hay derecho. Es decir, de la aceptación de la acción depende la cristalización del derecho. Su rechazo - por ende - significará la desaparición de este. En este punto tiene mucho que ver la claridad con la cual se presente ante el magistrado la clase o grupo afectado (certificación de la clase) o bien se caracterice el bien colectivo. En tal sentido, basta señalar lo sucedido con las sendas acciones de clase presentadas - a criterio de quien escribe, negligentemente- en virtud de los aumentos tarifarios en materia de energía(2), o bien - con carácter más reciente- respecto de cuestiones previsionales (3).

(2) In re "Abarca, Walter José y otros c/ Estado Nacional - Ministerio Energía y Minería y otro s/ Amparo ley 16.986" FLP 1319/2016, entre muchos otros.

(3) Juzgado Fed. Seg. Social No 9 - 03/03/2020, "Lezaun, Silvia Inés y Otro c. ANSES s/ amparos y sumarísimos". 
En el próximo apartado hablaré respecto a ciertas características que la Corte ha impreso al Proceso Ambiental, al cual definiré siempre como "colectivo" en virtud del bien afectado y siempre que hablemos de daño directo.

\section{Acciones de clase y proceso ambiental}

Las acciones de clase tienen larga tradición en el derecho norteamericano y han sido objeto de alabanzas por sus características: propenden a la economía procesal, colaboran con la concentración y el acceso a la justicia y —además- sirven para democratizar la jurisdicción con asuntos que de otro modo no hubiesen tenido lugar en los tribunales, disminuyendo los costos notablemente. También han sido criticadas debido al argumento contramayoritario en la revisión judicial de decisiones parlamentarias (planteado en su momento por Bickel) que se ve derribado - entre otros- en el precedente Carolene Products (4) y también por el lobby o presión del sector empresario. Desde su instauración en 1938, la Regla 23 sufrió una enmienda relevante en 1966 y otras de carácter menor en 1987, 1998, 2005 y 2009.

El máximo tribunal argentino dijo haber acogido dichas reglas en su precedente "Halabi" (5) y en virtud de una categoría existente en el artículo 43 de la Constitución Nacional, parafraseo mediante de los fallos "Siri" (6) y "Kot" (7) y echando mano a la operatividad de las garantías constitucionales. No obstante, si se examina cuidadosamente aquel precedente (y los subsiguientes en la materia) se notará que tal sentencia no es un mero trasplante o aplicación autómata de la mencionada Regla 23, toda vez que aquella prevé distintos mecanismos para su presentación, dependiendo de la materia y las circunstancias del caso y en el supuesto argentino solo se prevé el supuesto de encontrarse ante los llamados "intereses individuales homogéneos" señalados por el citado artículo 43 de la ley fundamental. En este sentido, "Halabi" solo adopta los requisitos fundamentales o generales de la Regla 23: numerosidad, cuestión fáctica común, tipicalidad y adecuada representación (Considerando 17), aunque sin distinguir entre materias como lo hace la regla norteamericana.

Por otra parte, no se trataría del mismo caso según se trate de esta categoría respecto de otras como ser la ambiental (bien colectivo por excelencia), excepto que se trate del daño ambiental denominado "indirecto" que afecta a una clase o

(4) U.S., S.C., "United States v. Carolene Products Co." 304 US 144 (1938).

(5) CSJN, “Halabi, Ernesto c. PEN - Ley 25.873 - dec. 1563/2004 s/ amparo ley 16.986", H.270.XLII, $24 / 02 / 2009$.

(6) CSJN, 27/12/1957, “Siri, Ángel”, 239:459 - JA 1958-II-478 60000002.

(7) CSJN, “Samuel Kot S.R.L.” - 05/09/1958 - La Ley 92, 626, AR/JUR/2/1958. 
grupo, tal como el mismo cuerpo jurisdiccional dejó entrever en "Mendoza" (8), "que más allá de lo expresado, cabe señalar con respecto a dicha pretensión que, si bien, eventualmente, podrían ser calificados como intereses individuales homogéneos, en razón de que podría haber un solo hecho ilícito que cause lesiones diferenciadas a los sujetos peticionantes, ello no surge de la demanda, en la medida en que, por el contrario, menciona diferentes supuestos de causación" (considerando 17, causa "Mendoza").

Luego, la Corte argentina emitió dos acordadas (9) a efectos de reglamentar los procesos colectivos en general, cuestión que aparece más como el asalto a una prerrogativa exclusiva del Congreso Nacional y no como un mero ordenamiento de los tribunales inferiores en virtud de la incompatibilidad existente entre los códigos de rito vigentes y las reglas propias de las acciones colectivas.

El proceso ambiental —que no fue regulado en la acordada 12/2016, reservada para acciones de clase propiamente dichas- aparece entonces con determinadas características que lo presentan como una acción sui generis dentro de los procesos o acciones colectivas en general: facultades especiales de los jueces, aplicación de reglas propias de la materia y sentencias con ejecución monitoread, son algunas de las notas particulares que la Corte reitera en sus precedentes.

\section{Los precedentes de la Corte argentina y las facultades del juez}

Entre las sentencias más resonantes de la Corte, sobre las cuales se ha escrito por demás (en honor a la brevedad me remito a ello) se encuentra el citado leading case "Mendoza". Dicho decisorio, que trató sobre un bien natural históricamente degradado, fue el puntapié para elaborar una doctrina propia del proceso ambiental. Luego de hacer referencia al daño indirecto (aquel que es causado al ser humano cuando se degrada al ambiente, también denominado "de rebote") el máximo tribunal argentino señaló la importancia que tiene la protección del ambiente y su sostenibilidad:

La tutela del ambiente importa el cumplimiento de los deberes que cada uno de los ciudadanos tienen respecto del cuidado de los ríos, de la diversidad de la flora y la fauna, de los suelos colindantes, de la atmósfera. Estos deberes son el correlato que esos mismos ciudadanos tienen a disfrutar de un ambiente sano, para sí y para las generaciones futuras, porque el daño que un individuo causa al bien colectivo se lo

(8) CSJN, "Mendoza Beatriz c. Estado Nacional y otros s/ daños y perjuicios", M.1569.XL, 20/06/2006.

(9) Acordada 32/2014 (Registro de acciones colectivas) y 12/16 (Reglamento de actuación). 
está causando a sí mismo. La mejora o degradación del ambiente beneficia o perjudica a toda la población, porque es un bien que pertenece a la esfera social y transindividual, y de allí deriva la particular energía con que los jueces deben actuar para hacer efectivos estos mandatos constitucionales (10) (Considerando 18, "Mendoza").

Los precedentes que siguieron a aquel, con algunas excepciones en criterio de quien suscribe, como ser el fallo "Ramírez" (11), en el cual el dictamen del procurador - que la Corte hizo suyo- omitió tratar la cuestión ambiental involucrada, fueron en alguna medida una reproducción de carácter docente hacia los tribunales inferiores, a efectos de que aquellos consideren las herramientas y facultades con las que cuentan ante un caso en el que se encuentra en juego la afectación al ambiente. Estas facultades no son meramente pretorianas, sino que se derivan de la aplicación armoniosa que hace la Corte del Artículo 41 de la Constitución Nacional y de la Ley general del ambiente No 25.675.

Entre otros, el fallo "Kersich" (12), que resulta en una comunión cuestiones ambientales y de consumo (trató sobre el consumo de agua contaminada con alto contenido de arsénico) y que aparece como una clara acción de clase, tiene importantes consideraciones sobre el carácter de director del juez cuando el ambiente se ve afectado. En efecto, la Corte señaló en aquel decisorio:

Que los jueces de la causa no aplicaron las reglas del proceso colectivo previsto en el artículo 43 de la Constitución Nacional, cuyas características principales y modalidades fueron enunciadas por esta Corte Suprema en el caso "Halabi" (Fallos: 332:111) y mantenidas consistentemente en los casos (...). Esta deficiencia se patentiza cuando el juez de primera instancia, pese a calificar al presente como amparo colectivo, recurrió a reglas procesales incompatibles con ese tipo de proceso, soslayando las consecuencias negativas que tal temperamento ocasionaría en el normal trámite de la causa. Máxime cuando la provincia de Buenos Aires dispone de normativa específica (con base en el artículo 20 de la Constitución Provincial, en especial, ley 13.928, con modificaciones introducidas por ley 14.192) que aplicada armoniosa y sistemáticamente, y de acuerdo con los principios rectores de la Ley general del ambiente, hubiese impedido la violación palmaria del de-

(10) El destacado me pertenece.

(11) CSJN, R. 1398.XLI. "Ramírez Juan Carlos c/ Entidad Binacional Yacyretá s/ Daños y Perjuicios" (2007).

(12) CSJN, "Kersich Juan Gabriel y otros c/ Aguas Bonaerenses S.A. y otros s/amparo". Fallo $42 / 2013(49-K)$. 
recho de defensa en juicio de la agencia estatal demandada (considerando 9, "Kersich").

Con el mismo sentido, la corte se refirió a la importancia del ambiente y del agua en particular:

En el campo de los derechos de incidencia colectiva es fundamental la protección del agua para que la naturaleza mantenga su funcionamiento como sistema y su capacidad de resiliencia. Por esta razón es que en muchos instrumentos internacionales se menciona la tutela del derecho al agua potable. En este sentido, la resolución A/RES/64/292, del 30/07/2010, de Naciones Unidas, declaró el derecho al agua potable y el saneamiento como derecho humano esencial para el pleno disfrute de la vida y de todos los derechos humanos. La "Convención sobre la Eliminación de todas las Formas de Discriminación contra la Mujer" (1979), artículo 14, párr. 2 y el Protocolo Adicional a la Convención Americana sobre Derechos Humanos, en materia de Derechos Económicos, Sociales y Culturales "Protocolo de San Salvador" del $17 / 11 / 1988$, predican que toda persona tiene derecho a un medio ambiente sano y a contar con los servicios básicos; la "Convención sobre los Derechos del Niño", artículo 24, 20 párr. (1989), exige a los Estados Partes que luchen contra las enfermedades mediante el suministro de agua potable salubre. De otro lado, es de recordar que en el mes de septiembre de 2000 los dirigentes de todos los países se comprometieron en la Cumbre del Milenio de las Naciones Unidas a reducir a la mitad para el afta 2015 la proporción de personas que carecían de acceso al agua potable o que no podían costearla. Y que en la Cumbre Mundial sobre el Desarrollo Sostenible de 2002, celebrada en Johannesburgo, se acordó un objetivo similar para reducir a la mitad, también para el año 2015, las cifras de personas sin saneamiento básico (...) (considerando 12, "Kersich").

Sobre este punto la Corte volvería en el litigio histórico entre la provincia de La Pampa y la provincia de Mendoza por el Río Atuel(13):

El ambiente no es para la Constitución Nacional un objeto destinado al exclusivo servicio del hombre, apropiable en función de sus necesidades y de la tecnología disponible, tal como aquello que responde a la voluntad de un sujeto que es su propietario.(...) En el sub examine el desentendimiento entre las provincias de La Pampa y Mendoza se

(13) CSJN, 243/2014 (50-L) ICS1, "La Pampa Provincia de c/ Mendoza Provincia de s/ uso de aguas". 
ha mantenido durante décadas, siendo necesario encontrar una eficaz canalización' racional de la disputa que evite escenarios de ahondamiento de las desavenencias; con mayor razón aun cuando en la causa se dirime una cuestión constitucional de la mayor relevancia, como lo es la preservación del ambiente y su sustentabilidad intergeneracional (artículo 41 de la Constitución Nacional), en el que se halla comprometido el interés general (doctrina del artículo 32 de la ley 25.675) (14) (Considerando 8, "La Pampa c. Mendoza").

En relación con las facultades, es interesante lo señalado en "Fundación Ciudadanos Independientes" (15): "que de tal manera, el tribunal como custodio que es de las garantías constitucionales, y con fundamento en la Ley General del Ambiente, en cuanto establece que 'el juez interviniente podrá disponer todas las medidas necesarias para ordenar, conducir o probar los hechos dañosos en el proceso, a fin de proteger efectivamente el interés general' (artículo 32, ley 25.675)" (Considerando 50, "Fundación Ciudadanos Independientes").

Entre las decisiones más recientes de la Corte se encuentra "Majul" (16), en el cual se produjo - nuevamente- tensión entre desarrollos inmobiliarios y ambiente (Muñoz, 2019) y allí se desarrollaron principios como el in dubio pro acqua o in dubio pro natura (que pueden también ser caracterizados como sub principios del precautorio) y que suponen que en caso de duda se debe estar en favor del ambiente y del agua, recurso vital para el ser humano. Desde el punto de vista de la Corte Argentina, el Tribunal Superior de Justicia de Entre Ríos, además de soslayar la correcta aplicación del principio precautorio (Ley general del ambiente 25.675, artículo $4^{\circ}$ ) omitió considerar el derecho a vivir en un ambiente sano, máxime cuando se encuentra en juego la protección de los humedales, en razón de los servicios ecosistémicos que prestan y que la propia constitución provincial protege.

\section{El caso España: un ejemplo comparado}

En Europa existe un evidenciado rechazo a la acción de clase, o al menos a su trasplante en las condiciones que propone el sistema norteamericano. Particularmente existen diferencias sobre la legitimación, y el manejo de casos en los cuales de daños a nivel masivo (Kocher, 2011). Sin perjuicio de lo expuesto, en

(14) El destacado me pertenece.

(15) CSJN, 121/2009, "Fundación Ciudadanos Independientes c/ San Juan y otros s/ acción ambiental meramente declarativa".

(16) CSJN, 714/2016/RH1, "Majul, Julio Jesús c/ Municipalidad de Pueblo General Belgrano y otros s/ acción de amparo ambiental". 
materias como derecho del consumidor y derecho ambiental la tendencia parece revertirse, siendo el diseño de procesos colectivos un importante desarrollo en la jurisprudencia comunitaria, así como en la de los miembros de la UE.

En España, como ejemplo del caso europeo puede señalarse que la legislación - y en una misma línea, la jurisprudencia- española y comunitaria no contemplan acciones de clase stricto sensu (es decir, aquellas acciones nacidas en Norteamérica - sin perjuicio de su origen histórico-, a tenor de la Regla 23), sino que, de manera detallada y restrictiva, comprende legitimación colectiva para la tutela de determinados bienes jurídicos, particularmente en la esfera civil para casos de consumo (por intermedio de la Ley de enjuiciamiento civil(17)), y en la órbita administrativa, para aquellas afectaciones al medio ambiente (a través de una profusa normativa, encabezada a nivel local por la Ley de responsabilidad medioambiental(18)).

Es menester señalar que, cuando se trata de medio ambiente, la concepción difiere de aquella que prevé la legislación argentina, tanto en la interpretación pretoriana que se hace de la disposición constitucional (conf. artículo 41-43 CN), así como también en referencia a las disposiciones de la Ley general del ambiente № 25.675, que contempla una definición amplia sobre el ambiente como bien colectivo (conf. artículo $2^{\circ}$, inc. a de la precitada ley).

En efecto, en la legislación y jurisprudencia española comparada, medio ambiente involucra solo a los recursos naturales (Rodríguez Betancor, 2011) tal como lo define la ley de responsabilidad medioambiental 26/2007.

Por otra parte, en cuestiones medioambientales (así como referidas a urbanismo, que trataría en parte también casos de afección al ambiente no-natural) el fuero es eminentemente administrativo (Pardo, 2008), con recursos frente a actos, omisiones y/o actuación de la administración en la órbita jurisdiccional del contencioso-administrativo (conf. Ley de responsabilidad medioambiental 26/2007), pero si se trata del daño indirecto provocado por la afectación al medio ambiente, dicha reclamación procederá por la vía ordinaria civil, donde se reclamarán los daños que tengan lugar por derecho. También existen acciones directas que pueden interponerse en el fuero contencioso-administrativo (por ejemplo, la denuncia de un delito ambiental; cuando hay afectaciones a la salud, etc.), sin necesidad de una actuación administrativa previa.

Efectuada dicha aclaración y retomando la línea referida a procesos colectivos en general, debe señalarse que los mismos no cuentan con un andamiaje único

(17) Ley 1/2000, de 7 de enero, de Enjuiciamiento Civil. BOE-A-2000-323.

(18) Ley 26/2007, de 23 de octubre, de Responsabilidad Medioambiental. BOE-A-2007-18475. 
(consumo, ambiente, discriminación, etc.), sino que dependerá de cada materia para evaluar la ley aplicable y la legitimación colectiva.

En este sentido, no cuentan con un dispositivo comunitario, constitucional o bien legislativo en general, que trate sobre derechos de incidencia colectiva, sin perjuicio de las acciones populares que reconocen de manera aislada algunas leyes. Asimismo, la interpretación de determinadas normas obrantes en la Constitución de España no cuenta con una operatividad efectiva, sino que dependen de actos legislativos reglamentarios. En dicha línea, el artículo $45 \operatorname{CE(19),~que~}$ dispone el derecho de disfrute al medio ambiente "adecuado para el desarrollo de la persona", así como también "la obligación de preservarlo", es interpretado doctrinal y jurisprudencialmente excluidos del amparo constitucional y se señala por tanto, que dicho precepto se encuadra como "principio Rector de la política social y económica" y que de acuerdo con el artículo $53 \mathrm{CE}$, "solo podrán ser alegados ante la jurisdicción ordinaria de acuerdo con lo que dispongan las leyes que los desarrollen" (Lozano Cutanda, 2010). La Jurisprudencia ha tenido una visión similar sobre el punto (20).

No obstante, que la constitución configure el medio ambiente como bien colectivo informador del ordenamiento no significa que su proclamación en el texto constitucional carezca de operatividad normativa. Es decir, también se ha señalado que no se trata de una "mera invitación" (21).

En cuanto a las competencias (Lasagabaster Herrarte et al., 2014) es interesante también señalar el reparto de estas entre los diferentes entes públicos involucrados, en particular en relación con el ambiente, legislación que debe trasponerse desde la Unión Europea (reglamentos, directivas y decisiones), así como la Competencia Exclusiva del Estado (ordenamiento estatal: artículo 149123 de la CE) para dictar legislación básica sobre protección del medio ambiente. Al respecto, las Comunidades Autónomas solo pueden dictar normas adicionales o complementarias de protección, respetando el piso o base determinado por la legislación estatal. Así también lo ha entendido el Superior Tribunal(22).

(19) Constitución Española. BOE-A-1978-31229.

(20) "No todos los preceptos constitucionales, sino exclusivamente aquellos a los que expresamente se refiere el artículo 53.2 de la Constitución, contienen principios o derechos susceptibles de protección a través del recurso de amparo". Tribunal Constitucional. Sentencia 4/1989 de 18 de enero y, en el mismo sentido, Sentencia 161/1987 de 27 de octubre.

(21) "No hay en la Constitución normas meramente programáticas, sino que todos sus preceptos encierran un mandato preciso que vincula en sus propios términos a todos los poderes constitucionales". Tribunal Constitucional Español. Sentencia del 2/2/1981.

(22) Entre otras, sentencia del Tribunal Constitucional STC 166/2002. 
En materia de procesos colectivos resulta menester señalar aspectos íntimamente vinculados, como ser: a) el acceso a la justicia o jurisdicción y b) la legitimación. Este tema ha sido largamente tratado en el ámbito de la Unión Europea (v.gr. Comunicación 2017/C 275/01 de la Comisión relativa al acceso a la justicia en materia medioambiental), a fin de armonizar distintas legislaciones y culturas jurídicas de los Estados parte. Se han emitido importantes Reglamentos y Directivas UE, como también en su rol interpretativo (conf. Tratado de Funcionamiento de la UE), el Tribunal de Justicia de la Unión Europea dictó sentencias donde precisa los requisitos de acceso a la justicia.

Con relación a bienes colectivos, el ambiente es por excelencia uno de los mejores ejemplos expositivos. En este aspecto, el acceso a la justicia resulta fundamental para poder hacer valer las disposiciones protectorias de dichos bienes, que, por ser de carácter público, en muchas ocasiones no encuentran debido resguardo, ya sea por interés, por falta de potestades (legitimación), o por los costos que implica el litigio ambiental, particularmente en lo relacionado a los aspectos probatorios.

Por ello, se ha hecho hincapié reiteradamente en el acceso público a la información, a la participación y al debido acceso a la jurisdicción (Razquin Lizarraga y Ruiz de Apodaca, 2007). En efecto, el Tratado de Aarhus (aprobado mediante decisión del Consejo 2005/370/CE) ha sido un importante apuntalamiento de dichos pilares, que los Estados parte debieron trasponer en sus ordenamientos (23). España hizo lo propio a través de la ley 27/2006(24). Dicha disposición contiene mecanismos para la participación ciudadana, el acceso a la información por parte del público - incluyendo un andamiaje procesal a tales efectos-, así como también una referencia (aunque escueta) al acceso a la justicia en cuestiones medioambientales.

En relación con la legitimación, puede concluirse que, si bien existen mecanismos asemejables a la acción de clase, en rigor de verdad la legitimación frente a pretensiones colectivas resulta acotada a asociaciones u ONG que cumplen los requisitos de ley, pero no ha individuos que componen una clase o grupo.

(23) Argentina suscribió el similar acuerdo de "Escazú" elaborado para América Latina y el Caribe. Ver Ruiz de Apodaca y Muñoz, 2019.

(24) Ley 27/2006 de 18 de julio, por la que se regulan los derechos de acceso a la información, de participación pública y de acceso a la justicia en materia de medio ambiente (incorpora las Directivas 2003/4/CE y 2003/35/CE). BOE-A-2006-13010. 


\section{Conclusión}

A modo de corolario puede señalarse que el proceso ambiental argentino es - a ojos del máximo tribunal nacional - un caso sui generis, que ostenta sus propias reglas y principios y donde las decisiones del juez son flexibles y no se encuentran estrictamente atadas a reglas pétreas.

Estas reglas que orientan al proceso ambiental como tal, diferenciado de la acción de clase norteamericana, la cual es reservada para atender a los llamados "intereses individuales homogéneos" en los que una causa fáctica común afecta a un grupo o clase, tiene origen en la interpretación pretoriana de la ley general del ambiente, norma que reglamenta el artículo 41 de la Constitución Nacional y que resulta directriz para las restantes normas de presupuestos mínimos.

Esa interpretación de la Corte da lugar en el caso ambiental a que el juez pueda hacer uso de reglas de mayor latitud a efectos de proteger el ambiente: las cuestiones ambientales no admiten restricciones en el acceso a la jurisdicción y su tratamiento y el juez ambiental aparece como un verdadero director del proceso, en el cual puede ordenar toda medida que considere necesaria para probar el daño ambiental a efectos de preservar a este bien jurídico.

En otros sistemas comparados, como por ejemplo el caso español, donde influye por supuesto el panorama europeo general en cuanto a procesos colectivos, la visión del ambiente resulta aún acotada. En efecto, a diferencia de nuestro sistema, el concepto de medio ambiente es particularmente restringido a recursos naturales, ocupándose el urbanismo de otras cuestiones relacionadas al entorno. Esta cuestión tiene efectos en cuanto a la jurisdicción aplicable y a la legitimación, solo reservada ante el daño ambiental para organizaciones no gubernamentales (v.gr. asociaciones debidamente conformadas) pero no para el resto de los ciudadanos. De aquí puede derivarse que, de los tres pilares del derecho ambiental comunitario de acuerdo con el Convenio de Aarhus, el acceso a la justicia sea el que menor avance evidenció.

En virtud de lo señalado - y a modo de corolario- puede decirse que el proceso ambiental argentino reviste ciertas características que lo hacen único en su género, contrastando la virtud de reconocer en el juez ambiental amplias facultades para la protección del bien ambiental así como la amplia legitimación para la ciudadanía, con cierta incertidumbre subsistente para los justiciables, en tanto existe aún - en razón de su aún joven desarrollo- necesidad de formación de magistrados inferiores respecto a la materia ambiental. En este sentido, he omitido tratar, en honor a la brevedad, aspectos relacionados a la necesidad - $\mathrm{o}$ node especializar los fueros como ocurre en algunos supuestos locales y regionales. 


\section{Bibliografía}

Bidart Campos, G. (2001). Tratado Elemental de Derecho Constitucional Argentino, tomos I y II. Buenos Aires: Editorial Ediar.

Cafferatta, N. (2014). Derecho ambiental en el Código Civil y Comercial de la Nación. Suplemento especial nuevo Código Civil y comercial. Cita online http:// www.laley.thomsonreuters.com/AR/DOC/3833/2014. Buenos Aires: Abeledo Perrot.

Freer, R. y Collins Perdue, W. (2005). Civil Procedure. Cases, materials and questions. Fourth edition. ISBN: 0-8205-6238-6. Estados Unidos: Editorial Lexis Nexis.

Fiss, O. (1996). La teoría política de las acciones de clase. Revista Jurídica de la Universidad de Palermo, año 1, № 1 (pp. 5-12). Buenos Aires: Universidad de Palermo. Recuperado de https://www.palermo.edu/derecho/publicaciones/pdfs/ revista_juridica/n1N1-Abril1996/011Juridica02.pdf

Giannini, L. (2015). La necesidad de una reforma integral de la Justicia colectiva. Revista Anales de la Facultad de Ciencias Jurídicas y Sociales. Buenos Aires: La Ley. Recuperado de http://sedici.unlp.edu.ar/bitstream/handle/10915/50665/ Documento_completo.pdf-PDFA.pdf?sequence $=1$

Gil Domínguez, A. (2004). Los derechos colectivos como derechos fundamentales. En G. Bidart Campos y A. Gil Domínguez (coords.), A una década de la reforma constitucional. Buenos Aires: Editorial Ediar.

Gidi, A. y Ferrer Mac-Gregor, E. (2003). Procesos colectivos. La tutela de los derechos difusos colectivos e individuales en una perspectiva comparada. México: Editorial Porrúa.

Hantke-Domas, M. (2011). Avances legislativos en gestión sostenible y descentralizada del agua en América Latina. Santiago de Chile: CEPAL. Recuperado de https://repositorio.cepal.org/bitstream/handle/11362/3942/ S2011130_es.pdf?sequence=1\&isAllowed=y

Kocher, E. (2011). Collective rights and collective goods: enforcement as collective interest. En J. Steele y W. Van Boom, Mass Justice. Challenges of representation and distribution (pp. 118-137). ISBN: 978-1-84980-506-3. Inglaterra: Edward Elgar Publishing Limited.

Lasagabaster Herrarte, I.; García Ureta, A. y Lascano Brotóns, I. (2007). Derecho Ambiental, Segunda Edición. Bilbao: Editorial Lete. 
Lazarus, R. (2004). The making of environmental law. Chicago: The University of Chicago Press.

Lee, R. y Stech R. (2011). Access to environmental justice in England and Wales: funding representation for court reviews of administrative action. En J. Steele y W. Van Boom, Mass Justice. Challenges of representation and distribution (pp. 138-190). ISBN: 978-1-84980-506-3. Inglaterra: Edward Elgar Publishing Limited.

Lorenzetti, R. (2008). Teoría General del Derecho Ambiental. Buenos Aires: La Ley.

Lorenzetti, R. (2010). Justicia Colectiva. Buenos Aires: Rubinzal-Culzoni.

Losardo, M. F. (2015). Creación pretoriana de las acciones de clase, la problemática de la objeción contramayoritaria al órgano judicial y los diferentes modos de superarla ante la mora del legislador. Revista Lecciones y Ensayos, № 94. Buenos Aires: Facultad de Derecho, Universidad de Buenos Aires.

Lozano Cutanda, B. (2007). Derecho Ambiental Administrativo. 8va. ed. revisada y actualizada. Madrid: Editorial Dykinson SL.

Morello, A. (1994). El proceso civil colectivo. Revista Themis, № 30. Lima: Editorial Pontificia Universidad Católica del Perú.

Nino, E.; Maurino, G. y Sigal, M. (2006). Las acciones colectivas. Buenos Aires: Editorial Abeledo Perrot.

Pardo, J. E. (2008). Derecho del medio ambiente. 2da. ed. Madrid: Editorial Marcial Pons.

Pardo, J. E. (2014). Lecciones de Derecho Administrativo. 4ta. ed. Madrid: Editorial Marcial Pons.

Peces Morate, J. E. (2017). La Justicia en el urbanismo y el medio ambiente iSe cumplen las sentencias? Revista de Derecho Urbanístico y Medio Ambiente. ISSN 1139-4978 (pp. 323-342). Madrid: Editorial RDU.

Peñalver I Cabré, A. (2013). Las pretensiones en el contencioso-administrativo para la efectiva protección de los intereses colectivos. Revista de Administración Pública, No 190 (pp. 149-194). ISSN: 0034-7639. Madrid.

Peñalver I Cabré, A. (2014). El derecho humano al medio ambiente y su protección efectiva. R.V.A.P., No. especial 99-100 (pp. 2333-2357). ISSN: 0211-9560. Madrid. 
Percival, R.; Schroeder, C.; Miller A. y Leape, J. (2006). Environmental Regulation. Law, Science and Policy. 5th ed. Estados Unidos: Aspen publishers.

Peyrano, J. y Eguren, M. (2007). La batalla por la entronización legal de la medida autosatisfactiva. JA IV-1450. Buenos Aires: Abeledo-Perrot.

Pino Miklavec, N. (2013). La tutela judicial administrativa de los intereses ambientales. Estudio comparativo de los ordenamientos español y argentino. Madrid: Thomson Reuters Aranzadi.

Plaza Martin, C. (2006). Derecho Ambiental de la Unión Europea. Valencia: Editorial Tirant lo Blanch.

Razquin Lizarraga, J. y Ruiz de Apodaca Espinosa, A. (2007). Información, Participación y Justicia en Materia de Medio Ambiente. Comentario sistemático a la Ley 27/2006 de 18 de Julio. Madrid: Thomson Reuters Aranzadi.

Rodríguez Betancor, A. (2014). Derecho Ambiental. Madrid: La Ley.

Ruiz de Apodaca Espinosa, A. y Muñoz, M. (2019). El acuerdo de Escazú. Una mirada comparada con la experiencia europea de Aarhus. Revista temas de derecho civil, persona y patrimonio. Buenos Aires: Erreius.

Salgado, J. M. (2013). Proyecto para legislar los procesos colectivos. Revista Derecho Privado, año II, No 7. Buenos Aires: Editorial MJDH.

Sanchiz Moreno, F. (2007). Guía sobre el acceso a la Justicia Ambiental. Convenio de AARHUS. España: Editorial Asociación para la Justicia Ambiental Elaw.

Sandre Mayo, M. J. (2018). Las acciones colectivas en defensa de los consumidores. Madrid: Thomson Reuters Aranzadi.

Santos Diez, R. y Rodríguez Castelao, J. (2002). Derecho Urbanístico. Manual para juristas y técnicos. 5ta. ed. España: Editorial el consultor.

Sola, J. V. (2007). Los derechos de incidencia colectiva. Buenos Aires: Academia Nacional de Ciencias Morales y Políticas.

Sola, J. V. (2010). Control Judicial de Constitucionalidad. Buenos Aires: Abeledo-Perrot.

Sola, J. V. (2010). Derecho Constitucional. Buenos Aires: Abeledo-Perrot.

Stiglitz, R. y Pizarro, R. (2009). Reformas a la ley de defensa del consumidor. Buenos Aires: La Ley. 
Vallespin Pérez, D. (2018). Litigios sobre consumo: especialidades procesales y acciones colectivas. ISBN: 978-84-9090-277-6. Madrid: Editorial Bosch.

Valls, M. (2000). Jurisprudencia Ambiental, Legitimación. Buenos Aires: Ugerman Editor.

Fecha de recepción: 31-03-2020 Fecha de aceptación: 12-07-2020 